\title{
El análisis crítico de la cuestión energética: internacionalización y competencias profesionales en Periodismo
}

\author{
María Teresa Mercado SÁEZ \\ Universidad CEU Cardenal Herrera \\ mmercado@uch.ceu.es \\ Sebastián SÁNCHEZ CASTILLO \\ Universitat de València \\ sebastian.sanchez@uv.es
}

Recibido: $26 / 11 / 2012$

Aceptado: 23/01/2013

\begin{abstract}
Resumen
Las universidades han de poner en valor las competencias que se adquieren en la titulación de Periodismo que procuran un conocimiento racional y crítico del presente. Un presente en el que la cuestión energética es clave en la contribución al desarrollo de los principios de protección medioambiental, como señala el Real Decreto 1393/2007. Por otro lado, uno de los objetivos fundamentales del Espacio Europeo de Educación Superior (EEES) es fomentar la internacionalización y la movilidad de los estudiantes, tanto en Europa, como con otras partes del mundo. Por ello, esta investigación ofrece los resultados de un primer análisis comparativo en torno al debate energético con estudiantes de Comunicación de España y Chile, donde han creado el Sistema Chileno de Transferencia de Créditos (STC) similar al European Credit Transfer (ECTS).
\end{abstract}

Palabras clave: Periodismo, educación, energía, competencias, internacionalización

\section{Critical Analysis of the Energy Issue: Internationalization and Professional Competences in Journalism}

\begin{abstract}
Universities must adapt the professional competences acquired in the Degree in Journalism, which are aimed at students' development of rational and critical knowledge of the present day. In these days energy is a key issue in our contribution to the development of principles for environmental protection, as pointed out by Spanish Royal Decree 1393/2007. On the other hand, one of the main aims of the European Higher Education Area is the promotion of student internationalization and mobility both in Europe and worldwide. With this purpose, this research contributes the results of a preliminary comparative analysis on the energy issue between Spanish and Chilean Communication students, as Chile has recently implemented the Chilean Credit Transfer System (CCTS), which is similar to the European Credit Transfer System (ECTS).

Keywords: Journalism, education, energy, competences, internationalization

\section{Referencia normalizada}

MERCADO SÁEZ, María Teresa y SÁNCHEZ CASTILLO, Sebastián (2013): "El análisis crítico de la cuestión energética: internacionalización y competencias profesionales en Periodismo". Estudios sobre el Mensaje Periodístico. Vol. 19, Núm. especial marzo, págs.: 309-318. Madrid, Servicio de Publicaciones de la Universidad Complutense.
\end{abstract}

Sumario: 1. Introducción. 2. Metodología. 3. Desarrollo. 4. Conclusiones. 5. Referencias bibliográficas. 


\section{Introducción}

La enseñanza del Periodismo en las universidades españolas en el entorno del Espacio Europeo de Educación Superior (EEES) y en el contexto social y profesional actual supone todo un reto que exige poner en valor las competencias que se adquieren en la titulación que procuran "un conocimiento racional y crítico del presente con la finalidad de que el estudiante pueda comprender la sociedad actual para después transmitirla", tal y como recoge el Libro Blanco de la ANECA (2005: 308).

Consideramos que uno de los aspectos más importantes a tener en cuenta para entender el mundo en el que vivimos es la cuestión energética, teniendo en cuenta que el Real Decreto 1393/2007, de 29 de octubre, por el que se establece la ordenación de las enseñanzas universitarias oficiales, señala que la formación en cualquier actividad profesional debe contribuir al conocimiento y desarrollo de los principios de solidaridad y de protección medioambiental, entre otros.

La demanda mundial de energía se incrementará a un ritmo del 1,5\% anual durante el periodo 2007- 2030, según datos del World Energy Outlook elaborado por la Agencia Internacional de la Energía. Así, el sistema actual no será capaz de satisfacer las demandas de la población, en un mundo en el que 1.400 millones de personas carecen de acceso a la energía moderna. Reconociendo este desafío, la Asamblea General de las Naciones Unidas proclamó 2012 Año Internacional de la Energía Sostenible para todos.

La cuestión energética se presenta así como un asunto central para gobiernos, empresas, científicos y organizaciones ecologistas. Sin embargo, ni los problemas energéticos ni los medioambientales aparecen, en las encuestas a la población española, entre las principales preocupaciones mencionadas espontáneamente por los entrevistados en los Barómetros del Centro de Investigaciones Sociológicas (CIS): citado en 0,6 por ciento de las respuestas frente al 79,3\% del paro o $49,4 \%$ de los problemas de índole económica. Al mismo tiempo, un $53,1 \%$ de la población se reconoce poco $(45,3 \%)$ o nada informado al respecto (CIS, 2012).

Los ciudadanos confían, como fuentes de información sobre medio ambiente, según Pérez-Díaz y Rodríguez (2008), sobre todo en los científicos (un 68\%) y en los ecologistas (61\%). Muestran poca o casi nula confianza en el gobierno (10\%), las empresas $(6 \%)$ y los partidos políticos (3\%). En este ranking de confianza, se incorpora a los medios de comunicación en el listado consiguiendo el tercer puesto $(25,5 \%)$, aunque en realidad la mayoría acceden a esos actores clave a través de los medios: el 84,9 por ciento de los ciudadanos españoles obtiene la información ambiental a través de los medios (CIS, 2012).

Centrándonos en el aspecto energético, el CIS no considera la energía o el consumo energético de manera explícita en el listado de temas ambientales que pueden preocupar a los encuestados en relación a los problemas ambientales en general. La cuestión se plantea de la siguiente manera: "De la lista que voy a leerle, ¿podría indicarme los cinco temas ambientales que más le preocupan?" (CIS, 2012:15). De los 11 temas, los cinco que resultan más importantes para los ciudadanos encuestados son la contaminación de los ríos, lagos y aguas subterráneas (71,6 \% de las respuestas), el aumento de la temperatura de la Tierra $(58,1 \%)$, el aumento de residuos $(52,2)$, el ago- 
tamiento de los recursos naturales $(50,3)$ y el uso de pesticidas y productos químicos en la agricultura $(44,4 \%)$. La producción de energía está en el centro del debate acerca del calentamiento global o la sobreexplotación de los recursos y en otros temas que no se han considerado tan preocupantes: problemas propios de las ciudades, el impacto de los medios de transporte actuales y nuestros hábitos de consumo.

Cuando se les pregunta sobre la cuestión energética, los sondeos muestran el importante desconocimiento de la mayoría en relación al grado de dependencia energética del exterior, países que nos proveen de petróleo, fuentes y costes de producción eléctrica, etcétera (Pérez-Díaz y Rodríguez, 2008).

El aspecto más analizado es el relacionado con el uso de la energía nuclear. Según los sondeos (Eurobarómetro Especial 297), España es uno de los países menos proclives a la energía nuclear aunque no hay consenso científico en la explicación de las causas de estas creencias y opiniones. Si la media europea se encuentra en un $44 \%$ de apoyo en España se sitúa en un 24\%. La oposición de los españoles hacia esta fuente de energía durante tres décadas se ha visto acompañada también de la percepción de los problemas energéticos de España en cuanto a escasez de recursos y dificultades para atender la demanda energética, sin que haya afectado a esta actitud contraria. Los españoles se oponen a que la alternativa sea la energía nuclear. La escasez de energía fue planteada ya en el estudio de 1990 del CIS, y el 62\% de los españoles declaraba que esto sería un problema en el futuro. De hecho, a partir de los años noventa, las preguntas introducidas en los estudios de este organismo intentan precisamente conocer la opinión de los españoles sobre si España tendrá o no dificultades, a años vista, para cubrir las necesidades de demanda de energía. Desde 1990, año en el que se recogía una opinión diversificada sobre esta cuestión, parece que la tendencia de la opinión española refleja una creciente consciencia de la dificultad a medida que transcurren los años: $36 \%$ en 1990 , $42 \%$ en el Barómetro de febrero de 1995 y 49\% en el de noviembre de 2007.

Desde los primeros estudios, los españoles parecen apostar por la energía solar como alternativa energética. Ya en el estudio de 1996 del CIS, un 42,8\% manifestaba que deberían realizarse mayores avances en el ámbito de la energía solar (junto con los trasplantes de órganos, 74,9\%). Un aspecto que ya aparecía en los de 1978 y 1990, donde un $88 \%$ estaba de acuerdo con que en España se debería potenciar la utilización de energía solar porque se disponía de muchas horas de sol, y que seguirá presente hasta hoy.

En Energía y Sociedad. Actitudes de los españoles antes los problemas de la energía y del medio ambiente (Pérez-Díaz y Rodríguez, 2008) aparece una significativa dualidad en cuanto a la percepción y evaluación de los problemas energéticos. Por una parte, no aparecen entre los problemas principales de España mencionados espontáneamente por los entrevistados (tampoco los medioambientales, como ocurría en los del CIS). Por otra, si se les pregunta específicamente por alguno de estos problemas, la magnitud que les asignan tiende a ser enorme.

En relación con las soluciones a los retos energéticos (conservación, eficiencia, suministro, marco regulador, etc.), la postura declarada de la mayoría de los españoles resulta bastante ajustada a los postulados principales del movimiento ecologista pero se observan algunas inconsistencias y sobre todo, en la práctica, la disposición a 
aceptar los sacrificios que implican estos postulados es mínima. En general, en la formulación de los juicios sobre los asuntos comunes y la manera de afrontarlos, los ciudadanos suelen echar mano de información procedente de diversos medios de comunicación y de distintos participantes centrales en la discusión pública (organizaciones sociales, gobierno, empresas, partidos políticos, etc.).

En este sentido, teniendo en cuenta que la mayoría de los ciudadanos se informan sobre esta cuestión a través de los medios, es necesario incidir en la necesidad de la especialización periodística para mejorar el tratamiento informativo en el área ambiental en general, y en la energética en particular. Así, en La integración de la educación ambiental en la formación periodística (Mercado y Sánchez, 2012) se presentaba la primera investigación que pretendía potenciar la reflexión crítica y activar el proceso enseñanza-aprendizaje en torno a la problemática ambiental relacionándola con la actualidad periodística y las rutinas productivas de los medios en las aulas de Periodismo.

Los resultados de las encuestas realizadas a 116 alumnos de Ciencias de la Comunicación en relación al debate en torno a la conveniencia o no del uso de la energía nuclear en el marco más amplio y complejo de la crisis energética vinculada al consumo, provocaron el interés en conocer qué opinarían estudiantes de otros países en el marco de uno de los objetivos fundamentales del Espacio Europeo de Educación Superior (EEES). Los factores esenciales que promueve esta reforma educativa son: conjunto de títulos universitarios homogéneos, enseñanza basada en ciclos (grado, master, doctorado), nuevas metodologías de enseñanza-aprendizaje, medición del aprendizaje por competencias y no exclusivamente por contenidos, la aparición del ECTS (European Credit Transfer System) para unificar la medición del esfuerzo realizado por el alumno en los sistemas universitarios de los países de la UE; y por último, la internacionalización de la universidad (Sierra, 2010). En este sentido, con la finalidad de fomentar la internacionalización y "la movilidad de los estudiantes, tanto dentro de Europa, como con otras partes del mundo" (Real Decreto 1393/2007), Chile fue el elegido teniendo en cuenta la importancia de los países latinoamericanos en España y la creación del Sistema Chileno de Transferencia de Créditos (STC) similar al ECTS.

\section{Metodología}

El objetivo principal de esta investigación es potenciar la reflexión crítica y analizar el proceso enseñanza-aprendizaje en torno a la problemática ambiental relacionándola con la actualidad periodística y las rutinas productivas de los medios en estudiante de periodismo de la Universidad CEU Cardenal Herrera (Valencia, España) y la Universidad de Maule en Talca (Chile). El reconocimiento del papel de los medios de comunicación como constructores de la realidad y como educadores informales en la sociedad actual inspira la acción educativa que se presenta como proyecto de innovación docente en la práctica universitaria.

En concreto, la integración de la Educación Ambiental en la formación periodística se inicia con el análisis de los conocimientos y creencias de los futuros comunicadores acerca del consumo energético o la conveniencia del uso de la energía nuclear. También se trata de comprobar los efectos sociales de los media en cuanto a la vin- 
culación práctico-cognitiva tras el consumo (o no) de información periodística sobre la catástrofe de Fukushima.

La lucha internacional contra el cambio climático había devuelto al debate público la discusión acerca de la conveniencia o no del uso de la energía nuclear teniendo en cuenta que las actividades relacionadas con la energía (procesado, transformación, consumo...) representan el $80 \%$ de las emisiones de $\mathrm{CO} 2$ a escala mundial. Así, en los últimos años se había producido un apoyo creciente al uso de la energía nuclear concretada en la activación de planes nucleares, la continuación de otros o la renovación de licencias de explotación de centrales en varios países. Este apoyo se veía frenado en seco tras la catástrofe de Fukushima que impactaba con fuerza en la opinión pública de todo el mundo. En España no lo hacía tanto como en los países asiáticos que lo vivieron de cerca pero el 18\% de las personas que se oponían en 2011 a la energía nuclear se pusieron en contra de ella tras el accidente. Así lo aseguraba una encuesta realizada por la consultora Ipsos en 24 países (El País, 25 de junio 2011). De media en todos ellos, un $26 \%$ de opositores a las nucleares lo son por Fukushima.

La finalidad última es por tanto la mejora de la calidad de la enseñanza universitaria para que el Periodismo contribuya a la protección medioambiental, tal y como se especifica en el RD 1393/2007 y al cambio de paradigma que supone el EEES, donde "los estudiantes son participantes activos en sus propios procesos de aprendizaje desde un punto de vista metacognitivo, motivacional y comportamental" (Zimmerman, 1989).

Consideramos que la problemática ambiental en sus múltiples facetas debe formar parte de este seguimiento de la actualidad y estar integrada en las actividades globales dirigidas a los futuros comunicadores, aquellos que tendrán la posibilidad de actuar como educadores informales desde los medios, yendo más allá de los simples añadidos de temas ambientales.

El método de investigación utilizado es el correlacional utilizando como herramienta para la recogida de datos la encuesta mediante la que se recogen y analizan una serie de datos de una muestra de casos representativa de una población o universo más amplio, del que se pretende explorar, describir, predecir y/o explicar una serie de características (Vinuesa, 2005:177).

La encuesta de conocimiento medioambiental fue presentado para su elaboración a 116 estudiantes universitarios españoles de la Universidad CEU Cardenal Herrera y 110 chilenos de la Universidad Católica de Maule, en Talcal

Tras la creación de un libro de códigos (Igartua, 2006) con las variables consideradas (procedentes de la encuesta experimental propuesta) se procedió al análisis correlacional multivariable. Para la analogía de ambos grupos de estudio y comprobar si existen diferencias significativas entre las medias, así como los tratamientos comparados distintos se ha empleado el análisis de regresión lineal múltiple, R y R2 ajustado y ANOVA, así como los coeficientes Beta (coeficientes de regresión parcial estandarizados). Se ha empleado como software SPSS v.17.

\section{Desarrollo}

Los datos se han obtenido considerando como variable dependiente o criterio el país de origen de los alumnos (España o Chile), y como variables independientes o pre- 
dictoras cada una de las variables que componen la encuesta. Según se desprende de la tabla 1, la correlación entre el país de origen y las variables definidas ha resultado ser ,707 (con una correlación perfecta el valor de R será 1) y R2 corregida ,404.

\section{Tabla 1. Resumen de modelo $R$}

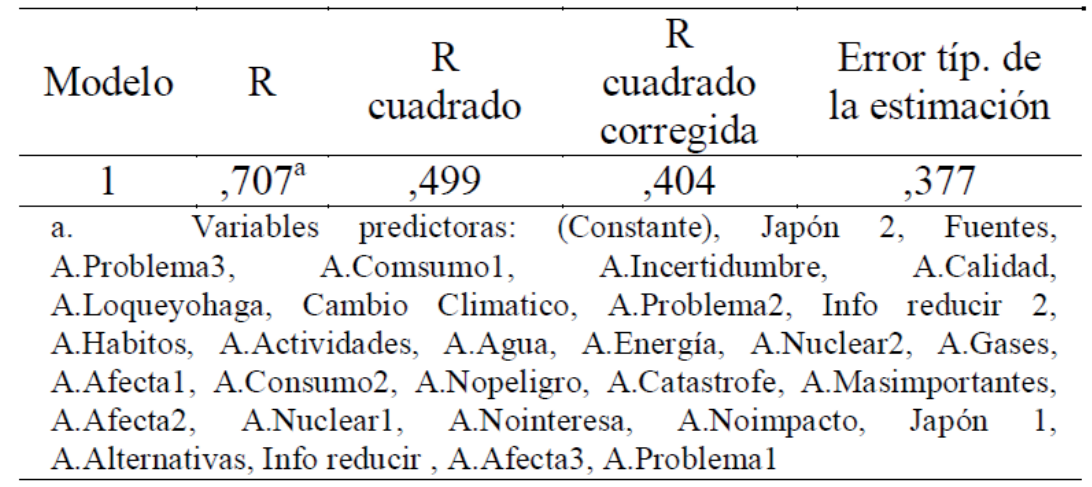

\section{Fuente: (Elaboración propia)}

El modelo de regresión hipotetizado (Igartua, 2006: 677) ha resultado estadísticamente significativo, ya que los valores de F de ANOVA [F $(30,158)=5,254, \mathrm{p}<, 001]$, que indica que la relación o dependencia entre la variable criterio y el conjunto de predictores es estadísticamente significativa, ver tabla 2.

Tabla 2. Correlación ANOVA ${ }^{\mathbf{b}}$

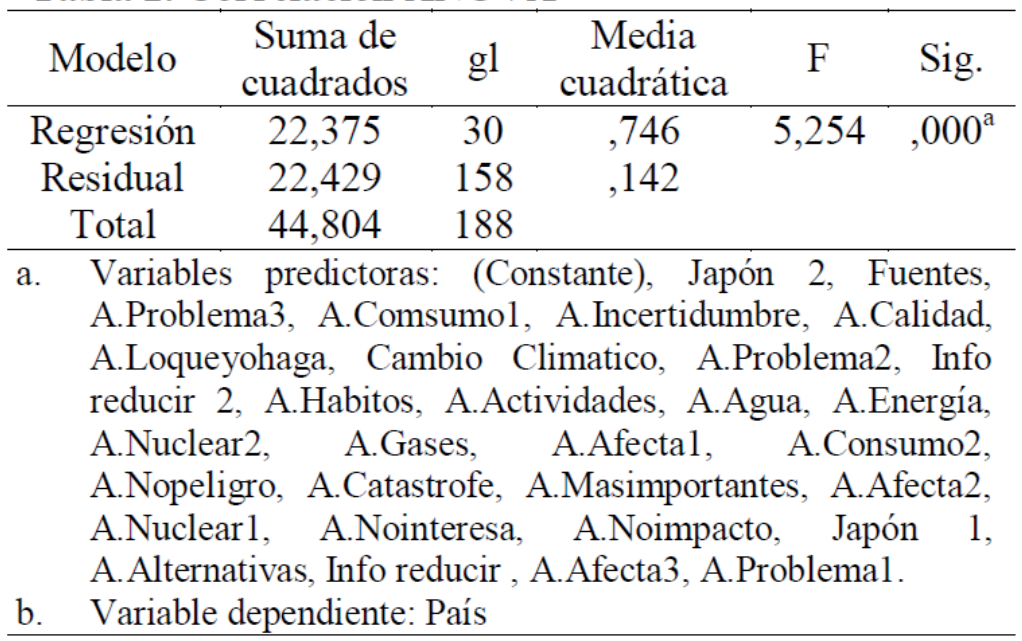

\section{Fuente: (Elaboración propia)}

De esta forma, a la pregunta ¿cuál es, a su juicio, el principal problema que existe actualmente en España y en Chile?, la respuesta ha resultado ser claramente dependiente del país de origen del estudiante, $[\beta=, 449, \mathrm{t}(158)=6,451, \mathrm{p}<, 001]$. También el segundo problema se ha visto correlacionado, $[\beta=, 133, \mathrm{t}(158)=2,150, \mathrm{p}<, 033]$. 
El 62,06\% de los encuestados en España respondía que el principal problema del país era el paro mientras que en Chile, los estudiantes señalaban la educación $(31,50$ $\%)$, también referido como el problema que más les afecta (54,79\%).

En ambos países, los estudiantes se muestran preocupados al preguntarles de manera explícita si creen que la escasez de energía puede ser uno de los problemas más importantes que tendrán que afrontar los países desarrollados (82,75 por ciento de los españoles y 91,78 de los chilenos respondieron afirmativamente).

Sobre la cuestión de si "la energía nuclear es competitiva y garantiza el suministro eléctrico", también se han descubierto una relación según el país, $[\beta=-, 272, \mathrm{t}$ $(158)=-4,275, p<, 001]$. Solo el $14,65 \%$ de los estudiante españoles se mostraba de acuerdo con esta afirmación. La cifra asciende al 54,79 por ciento en las respuestas de los chilenos. En la misma variable "la energía nuclear debe desaparecer porque genera residuos altamente radiactivos", en este caso una correlación con menos intensidad $[\beta=, 125, \mathrm{t}(158)=2,009, \mathrm{p}<, 046]$. Al preguntarle acerca de qué fuente de energía debería utilizarse menos (carbón, gas, petróleo, nuclear, renovables), el 53,44 por ciento de los españoles cita la nuclear. Los chilenos prefieren dejar de utilizar el gas, petró-

Tabla 3. Coeficientes ${ }^{a}$. Variable dependiente "País"

\begin{tabular}{|l|c|c|c|}
\hline \multicolumn{1}{|c|}{ Variable } & Beta & $\mathbf{t}$ & Sig. \\
\hline A.Problema1 &, 449 & 6,451 &, 000 \\
\hline A.Problema2 &, 133 & 2,150 &, 033 \\
\hline A.Problema3 &, 022 &, 348 &, 728 \\
\hline A.Afecta1 &,- 026 &,- 397 &, 692 \\
\hline A.Afecta2 &,- 002 &,- 034 &, 973 \\
\hline A.Afecta3 &, 011 &, 166 &, 868 \\
\hline A.Energía &,- 093 & $-1,482$ &, 140 \\
\hline A.Actividades &,- 080 & $-1,303$ &, 194 \\
\hline Fuentes &, 004 &, 066 &, 947 \\
\hline Cambio Climatico &, 069 & 1,109 &, 269 \\
\hline A.Alternativas &, 008 &, 113 &, 910 \\
\hline A.Nuclear1 &,- 272 & $-4,275$ &, 000 \\
\hline A.Nuclear2 &, 125 & 2,009 &, 046 \\
\hline A.Comsumo1 &,- 085 & $-1,351$ &, 178 \\
\hline A.Consumo2 &,- 011 &,- 173 &, 863 \\
\hline A.Calidad &,- 202 & $-3,357$ &, 001 \\
\hline A.Agua &, 003 &, 047 &, 962 \\
\hline A.Gases &,- 016 &,- 267 &, 790 \\
\hline A.Incertidumbre &, 000 &,- 003 &, 997 \\
\hline A.Catastrofe &,- 094 & $-1,506$ &, 134 \\
\hline A.Habitos &, 024 &, 406 &, 686 \\
\hline A.Loqueyohaga &,- 008 &,- 128 &, 898 \\
\hline A.Nointeresa &,- 043 &, 047 &, 508 \\
\hline A.Noimpacto &, 125 & 1,958 &, 052 \\
\hline A.Masimportantes &,- 016 &,- 248 &, 804 \\
\hline A.Nopeligro &,- 028 &,- 442 &, 659 \\
\hline Info reducir &,- 013 &,- 195 &, 845 \\
\hline Info reducir 2 &,- 123 & $-1,849$ &, 066 \\
\hline Japón 1 &, 004 &, 065 &, 948 \\
\hline Japón 2 &, 032 &, 463 &, 644 \\
\hline Fuen & &
\end{tabular}

Fuente: (Elaboración propia) leo y carbón $(64,38 \%)$.

A la afirmación "nuestra calidad de vida mejoraría si gastáramos menos electricidad, gas o gasolina", se ha contestado también de forma dependiente, $[\beta=-, 202, \mathrm{t}(158)=$ $-3,357, \mathrm{p}<, 001]$. Un 72,6 por ciento de los estudiantes de Chile estaban muy de acuerdo, un porcentaje bastante elevado y muy superior al $56,02 \%$ español. En el mismo sentido, el $24,65 \%$ de los chilenos estaba muy de acuerdo en que el consumo de electricidad no afecta a la contaminación frente al $10,34 \%$ de los estudiantes en nuestro país.

De la misma forma, aunque ligeramente tendente, se muestra la expresión "lo que yo haga no tiene impacto si las empresas o industrias contaminantes no hacen más esfuerzos por proteger el medio ambiente", $[\beta=, 125, \mathrm{t}(158)=-1,958, \mathrm{p}<$ ,052]. Por último, a la pregunta 
referente a la información para reducir el consumo de energía, ¿podría decirme por qué medio ha recibido esta información? (medios, contactos personales, folletos de instituciones, charlas o conferencias, libros otros), se ha descubierto una mínima dependencia entre estos dos países, es decir que los estudiantes han recibido la información desde medios distintos, $[\beta=-, 123, \mathrm{t}(158)=-1,849, \mathrm{p}<, 066]$.

El $71,55 \%$ de los estudiantes españoles respondieron afirmativamente frente al $82,19 \%$ de respuestas afirmativas de los chilenos que en su mayoría recibió la información de los medios de comunicación (un 95,89\%), porcentaje menos significativo en el caso de los españoles: el 77,58\%, (ver tabla 3).

\section{Conclusiones}

Los resultados de la primera pregunta de la encuesta acerca del principal problema del país, no hacen otra cosa que demostrar la distinta coyuntura social. Mientras en España, el paro acecha a un número considerable de familias, en Chile la educación es una de las principales preocupaciones.

En 2011, el movimiento estudiantil chileno protestó en la calle pidiendo una educación pública gratuita y de calidad. En agosto de 2012, de nuevo salía a la calle dando "la mayor muestra de fuerza de este año con una marcha masiva y pacífica que solamente en Santiago ha reunido a unas 150.000 personas este martes en la Alameda, la principal avenida de la ciudad, según la cifra entregada por la Confederación de Estudiantes de Chile (Montes, El País, 2012). La Marcha Nacional por la Educación, que fue convocada por estudiantes de secundaria, universitarios, profesores y sindicatos, coronaba un mes marcado por las paralizaciones académicas, tomas de colegios y facultades y las manifestaciones.

De acuerdo a un sondeo de Imaginación y Radio Cooperativa, recogido por la corresponsal de El País, ocho de cada 10 chilenos apoya a las demandas estudiantiles, aunque el $67,3 \%$ está en desacuerdo con las tomas. El mismo estudio de opinión desvela que el 68,3\% de la población, sin embargo, está en desacuerdo con los desalojos de colegios y universidades que ha llegado a cabo el Gobierno para impedir que las actividades académicas se paralicen como en 2011.

Respecto al tema central de la investigación, los estudiantes chilenos se muestran mucho más de acuerdo que los españoles respecto a la competitividad de la energía nuclear aunque acercan posiciones en cuanto a que debería desaparecer debido a la peligrosidad de los residuos radioactivos que genera.

La energía nuclear es un tema de debate en Chile, un país deficitario desde el punto de vista energético cuyo desarrollo se puede ver comprometido si no logra establecer nuevas fuentes de abastecimiento. La posibilidad de instalar centrales nucleares surgía en el horizonte y se establecieron acuerdos con Francia para el desarrollo de la minería de uranio. En marzo de 2011 la Comisión Chilena de Energía Nuclear firmaba un acuerdo sobre cooperación en energía nuclear con el Gobierno norteamericano. El acuerdo trata fundamentalmente de la capacitación y entrenamiento de ingenieros y profesionales chilenos, pero fijaría también los canales para la eventual instalación de una planta nuclear de producción eléctrica, lo que provoca una fuerte controversia en medios de la oposición política y en los grupos ambientalistas. 
Como en el resto del mundo, la catástrofe de Fukushima ha frenado estas iniciativas. El ministro de Minería y Energía, Laurence Golborne, que en las primeras horas tras el terremoto y el tsunami en Japón difundió mensajes a través de twitter defendiendo las centrales nucleares, terminaba reconociendo que "lo ocurrido va afectar evidentemente la discusión a futuro" (Gallego-Díaz, El País, 14 marzo 2011). Sobre todo teniendo en cuenta que Chile es un país sísmico, que supera fuertemente los índices medios de riesgo de sufrir un terremoto de alta intensidad, como el que padeció en febrero de 2010. De hecho, los lugares estudiados por la Asociación Profesional de Ingenieros de Chile para la instalación de nuevas centrales todos ellos en la costa pacífica fueron afectados por el tsunami japonés, a más de 14.000 kilómetros del epicentro. Más de la mitad de los encuestados en ambos países reconocieron haber cambiado de su forma de pensar sobre el uso de la energía nuclear tras lo sucedido en Japón.

Un año antes de Fukushima, el 27 de febrero de 2010, un terremoto de 8,8 grados de intensidad en la escala de Richter, el más fuerte en 50 años en Chile, sacudió la zona central y sur del país y fue seguido de una serie de maremotos. 524 personas perdieron la vida. El seísmo más violento registrado hasta ahora, con una magnitud de 9,5, también se produjo en Chile, al sur de Concepción, en 1960.

En algunas de las cuestiones en las que los datos muestran divergencias según el país, los estudiantes chilenos parecen más concienciados en el excesivo consumo energético y al mismo tiempo en la propia responsabilidad ante la crisis ambiental. Parecen menos proclives a responsabilizar de solucionar los problemas de contaminación que han generado.

\section{Referencias bibliográficas}

ANECA (2005): Libro Blanco. Títulos de grado en Comunicación. Madrid, Agencia Nacional de Evaluación de la Calidad y Acreditación.

CIS (1993): Energía Nuclear. Estudio 2.074.

CIS (1996): Ecología y Medio Ambiente. Estudio 2.209.

CIS (2007): Ecología y Medio Ambiente III. Estudio 2.682.

CIS (2012): Barómetro de Septiembre 2012. Estudio 2.954.

GALLEGO-DÍAZ, Soledad (2011): "El debate nuclear se aviva en Chile", en El País, 14 de marzo de 2011. Sección Internacional: http://internacional.elpais.com/internacional/2011/03/14/actualidad/1300057220_850215.html [fecha de consulta: 31 julio 2012]

IGARTUA, Juan José (2008): Métodos cuantitativos de investigación en Comunicación. Barcelona, Bosch.

MERCADO, Ma Teresa y SÁNCHEZ, Sebastián (2012): "La integración de la educación ambiental en la formación periodística como proyecto de innovación docente". Vivat Academia, n 117, pp. 936-963.

MONTES, Rocío (2012): "El movimiento estudiantil en Chile recupera su fuerza", en El País, 29 de agosto de 2011. Sección Internacional: http://internacional.el- 
pais.com/internacional/2012/08/29/actualidad/1346193931_225376.html [fecha de consulta: 29 agosto 2012]

PÉREZ-DÍAZ, Víctor y RODRÍGUEZ, Juan Carlos (2008): Energía y Sociedad. Actitudes de los españoles antes los problemas de la energía y del medio ambiente. Madrid, Club Español de la Energía.

SIERRA, Javier (2010): "Competencias profesionales y empleo en el futuro del periodista: el caso de los estudiantes de periodismo de la Universidad Abat Oliba CEU”. RED. Revista de Educación a Distancia. Número 1: http://www.um.es/ead/reddusc/1 [fecha de consulta: 10 septiembre de 2012].

ZIMMERMAN, Barry (1989): "A social-cognitive view of self-regulated academic learning”. Journal of Educational Psychology, 81, pp. 329-339.

\section{Dra. María Teresa MERCADO SÁEZ}

Universidad CEU Cardenal Herrera. Facultad de Humanidades y Ciencias de la Comunicación

Doctora en Ciencias de la Información (UCM). Profesora de Periodismo. Directora del Instituto CEU de Disciplinas Económicas, Ambientales y Sociales (IDEAS). Investigadora Principal del Grupo 'Sostenibilidad y Periodismo Especializado'.

mmercado@uch.ceu.es

\section{Dr. Sebastián SÁNCHEZ CASTILLO}

Facultat de Filologia, Traducció i Comunicació.

Doctor en Comunicación Audiovisual (UV). Profesor Asociado en el Departamet Teoria dels Llenguatges i Ciències de la Comunicació de la Facultat de Filologia, Traducció i Comunicació, Universitat de València.

sebastian.sanchez@uv.es 